УДК 517.957:517.548

\author{
SOLUTIONS OF THE DIFFERENTIAL INEQUALITY \\ WITH A NULL LAGRANGIAN: HIGHER INTEGRABILITY \\ AND REMOVABILITY OF SINGULARITIES. I ${ }^{1}$
}

\author{
A. A. Egorov
}

Dedicated to Academician Yuri Grigor'evich Reshetnyak on the occasion of his 85th birthday

\begin{abstract}
The aim of this paper is to derive the self-improving property of integrability for derivatives of solutions of the differential inequality with a null Lagrangian. More precisely, we prove that the solution of the Sobolev class with some Sobolev exponent slightly smaller than the natural one determined by the structural assumption on the involved null Lagrangian actually belongs to the Sobolev class with some Sobolev exponent slightly larger than this natural exponent. We also apply this property to improve Hölder regularity and stability theorems of [19].
\end{abstract}

Mathematics Subject Classification (2000): 30C65 (primary), 35F20, 35A15, 35B35, $26 \mathrm{~B} 25$ (secondary).

Key words: null Lagrangian, higher integrability, self-improving regularity, Hölder regularity, stability of classes of mappings.

\title{
Introduction
}

In this paper and [22] we establish higher integrability and removability properties of solutions $v: V \rightarrow \mathbb{R}^{m}, V \subset \mathbb{R}^{n}$, of the following inequality

$$
F\left(v^{\prime}(x)\right) \leqslant K G\left(v^{\prime}(x)\right)+H(x) \quad \text { a. e. } \quad V
$$

constructed by means of a continuous function $F: \mathbb{R}^{m \times n} \rightarrow \mathbb{R}$, a null Lagrangian $G: \mathbb{R}^{m \times n} \rightarrow \mathbb{R}$, a measurable function $H: V \rightarrow \mathbb{R}$, and a constant $K \geqslant 1$. Here $v^{\prime}(x)$ denotes the differential of $v$ at $x \in V$. In the case $H(x) \equiv 0$ this inequality has the form

$$
F\left(v^{\prime}(x)\right) \leqslant K G\left(v^{\prime}(x)\right) \quad \text { a.e. } V \text {. }
$$

In [19], the author has obtained some results on closure of sets of solutions to (2) with respect to the local convergence in the Lebesgue space and their Hölder regularity (for example, see

(C) 2014 Egorov A. A.

${ }^{1}$ The study was supported by a grant from the Russian Foundation for Basic Research, project 14-0100768, the State Maintenance Program for the Leading Scientific Schools of the Russian Federation, grant № NSh-2263.2014.1, and the Integration Grant of the Siberian Division of the Russian Academy of Sciences, 2012 , № 56 . 
[19, Theorems 7 and 8]). Using these results, the author has proved the theorems ([19, Theorems 1 and 3-6]) on stability of the class of solutions to the equation

$$
F\left(u^{\prime}(x)\right)=G\left(u^{\prime}(x)\right) \quad \text { a. e. } x \in V
$$

(also see $[16-18])$.

Our main results are analogs of the well-known higher integrability and removability theorems for mappings with bounded distortion (quasiregular mappings). A mapping $v \in$ $W_{\text {loc }}^{1, n}\left(V ; \mathbb{R}^{n}\right)$ of an open set $V \subset \mathbb{R}^{n}$ is an (sense-preserving) mapping with $K$-bounded distortion, $K \geqslant 1$, if $v$ satisfies the distortion inequality

$$
\left|v^{\prime}(x)\right|^{n} \leqslant K \operatorname{det} v^{\prime}(x) \quad \text { a. e. } V
$$

where $\left|v^{\prime}(x)\right|$ is the operator norm of the matrix $v^{\prime}(x)$. If, in addition, $v$ is topological, then $v$ is $K$-quasiconformal. The distortion inequality is the particular case of (2) with the following functions $F\left(v^{\prime}(x)\right)=\left|v^{\prime}(x)\right|^{n}$ and $G\left(v^{\prime}(x)\right)=\operatorname{det} v^{\prime}(x)$. The theory of quasiconformal mappings and mappings with bounded distortion is the key part of modern geometric analysis which has many diverse applications (for example, see monographs $[2,6,10,30,31,32,36,41,45,46,49,52,53,58,59,60,61,62,63,68,69]$ and the bibliography therein).

The higher integrability for planar mappings with bounded distortion was established by B. Bojarski $[8,9]$. More precisely, he proved that there exists an exponent $\bar{p}(2, K)>2$ such that mappings with $K$-bounded distortion (a priori in $W_{\text {loc }}^{1,2}$ ) belong to $W_{\text {loc }}^{1, s}$ for every $s<\bar{p}(2, K)$. F. W. Gehring [28] has extended this result to $n$-dimensional quasiconformal mappings. The existence of an exponent $\bar{p}(n, K)>n \geqslant 3$ such that all $n$-dimensional mappings with $K$-bounded distortion lie in $W_{\text {loc }}^{1, s}$ for every $s<\bar{p}(n, K)$ was obtained by A. Elcart and N. G. Meyers [54] and, independently, by Yu. G. Reshetnyak [57] (also see [29, $50,51,58,59,60,61,62])$. Moreover, Yu. G. Reshetnyak [57] has established that $\bar{p}(n, K) \rightarrow$ $\infty$ as $K \rightarrow 1$ (also see [33]). For $n=2$ this result was obtained by O. Lehto [48]. The higher integrability result has a dual version. In two papers, T. Iwaniec and G. Martin [35] (for even dimensions) and T. Iwaniec [34] (for all dimensions) have proved that there exists an exponent $1<\underline{p}(n, K)<n$ such that if a mapping $v \in W_{\text {loc }}^{1, p}$ with some $p>\underline{p}(n, K)$ satisfies inequality (4), i. e. $v$ is a weakly quasiregular mapping, then $v$ belongs to $W_{\text {loc }}^{1, s}$ for every $s<\bar{p}(n, K)$ (also see [23, 36, 37] and for $n=2$ the monograph [49]). Here the word "weakly" means that the Sobolev integrable exponent $p$ of $v$ may be smaller then the dimension $n$. In this case, $\operatorname{det} v^{\prime}(x)$ need not be locally integrable. Thus the natural exponent for the distortion inequality is the dimension $n$. K. Astala [1] proved that $p(2, K)=2 K /(K+1)$ and $\bar{p}(2, K)=2 K /(K-1)$ are the sharp exponents for higher integrability of planar mappings with bounded distortion (also see [2]).

Also, higher integrability results have been established for the following mapping classes: the classes of mappings that are close to multidemensional holomorphic mappings [39, 40, 41]; the classes of mappings that are close to solutions of linear elliptic partial differential equations $[7,14,41]$; the classes of quasihomoteties [64, 65]; the classes of quasiregular mappings of several $n$-dimensional variables $[12,13,14]$; the classes of weakly $\left(K_{1}, K_{2}\right)$-quasiregular mappings [24, 27, 67]; the classes of degenerate weakly $\left(K_{1}, K_{2}\right)$-quasiregular mappings [26]; the classes of weakly $\left(K_{1}, K_{2}(x)\right)$-quasiregular mappings of several $n$-dimensional variables [25]; and a series of other calsses [41-43]. Mappings of these classes, as mappings with bounded distortion, can be considered as solutions to (1) with specific functions $F, G$, and $H$ (some examples of such considerations can be found in [19, §2]). Our main higher integrability 
result (Theorem 2.1) contain (either partially, or fully, or in an improved form) some of the known results on higher integrability for mappings of these classes. We also apply this result to improve the above-mentioned theorems on Hölder regularity and stability. In this paper, as in [16-19], we develop approaches and methods used for investigations of mappings with bounded distortion to study properties of solutions to (1). Some results of this paper have been announced in $[20,21]$.

The main aim of this paper is to prove Theorem 2.1 on higher integrability of solutions to (1). Applying this theorem, in the next paper [22], we establish a result on removability of singularities for solutions to (1). Also, in [22], using the Hodge decomposition theory developed by T. Iwaniec and G. Martin [34-36], we derive integral estimates for minors of Jacobian matrixs. These estimates have independent interest. In this paper they are used in the proof of Theorem 2.1.

This paper is organized as follows. In $\S 1$ we give the basic notation and terms. The main results are stated in $\S 2$. In $\S 3$ we expose some auxiliary lemmas. The proof of the higher integrability theorem (Theorem 2.1 is given in $\S 4$ ).

\section{Notation and Terminology}

Let $A$ be a set in $\mathbb{R}^{n}$. The topological boundary of $A$ is denoted by $\partial A$. The diameter of $A$ is defined as $\operatorname{diam} A:=\sup \{|x-y|: x, y \in A\}$. The outer Lebesgue measure of $A$ is denoted by $|A|$. We use the symbol $\operatorname{dim}_{H} A$ for the Hausdorff dimension of $A$.

The set $\mathbb{R}^{m \times n}:=\left\{\zeta=\left(\zeta_{\mu \nu}\right)_{\substack{\mu=1, \ldots, m \\ \nu=1, \ldots, n}}: \zeta_{\mu \nu} \in \mathbb{R}, \mu=1, \ldots, m, \nu=1, \ldots, n\right\}$ consists of all

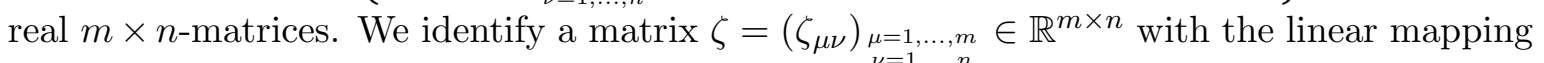
$\left(\zeta_{1}, \ldots, \zeta_{m}\right): \mathbb{R}^{n} \rightarrow \mathbb{R}^{m}$, where $\zeta_{\mu}(x):=\sum_{\nu=1}^{n} \zeta_{\mu \nu} x_{\nu}, \mu=1, \ldots, m, x=\left(x_{1}, \ldots, x_{n}\right) \in \mathbb{R}^{n}$. The operator norm in $\mathbb{R}^{m \times n}$ is defined as $|\zeta|:=\sup \left\{|\zeta(x)|: x \in \mathbb{R}^{n},|x|<1\right\}$. The number of $k$-tuples of ordered indices in $\Gamma_{n}^{k}:=\left\{I=\left(i_{1}, \ldots, i_{k}\right): 1 \leqslant i_{1}<\cdots<i_{k} \leqslant\right.$ $\left.n, i_{\varkappa} \in\{1, \ldots, n\}, \varkappa=1, \ldots, k\right\}$ equals the binomial coefficient $\left(\begin{array}{l}n \\ k\end{array}\right):=\frac{n !}{k !(n-k) !}$. Given $x \in \mathbb{R}^{n}$ and $I \in \Gamma_{n}^{k}$, we put $x_{I}:=\left(x_{i_{1}}, \ldots, x_{i_{k}}\right) \in \mathbb{R}^{k}$. If $I \in \Gamma_{n}^{k}$ and $J \in \Gamma_{m}^{k}$, then $\operatorname{det}_{J I} \zeta:=\operatorname{det}\left(\begin{array}{ccc}\zeta_{j_{1} i_{1}} & \cdots & \zeta_{j_{1} i_{k}} \\ \vdots & \ddots & \vdots \\ \zeta_{j i_{1}} & \cdots & \zeta_{j_{k} i_{k}}\end{array}\right)$ is the $k \times k$-minors of the matrix $\zeta \in \mathbb{R}^{m \times n}$. For $\varepsilon>-k$ we put $|\zeta|^{\varepsilon} \operatorname{det}_{J I} \zeta=0$ at $\zeta=0$.

The Jacobian matrix of $u=\left(u_{1}, \ldots, u_{m}\right): U \subset \mathbb{R}^{n} \rightarrow \mathbb{R}^{m}$ at a point $x \in U$ is the matrix $u^{\prime}(x):=\left(\frac{\partial u_{\mu}}{\partial x_{\nu}}(x)\right)_{\substack{\mu=1, \ldots, m \\ \nu=1, \ldots, n}}$. If $I \in \Gamma_{n}^{k}$ and $J \in \Gamma_{m}^{k}$ then $\frac{\partial u_{J}}{\partial x_{I}}(x)=\frac{\partial\left(u_{j_{1}}, \ldots, u_{j_{k}}\right)}{\partial\left(x_{i_{1}}, \ldots, x_{i_{k}}\right)}(x):=\operatorname{det}_{J I} u^{\prime}(x)$ and $\partial_{I} u_{\mu}(x):=\left(\frac{\partial u_{\mu}}{\partial x_{i_{1}}}(x), \ldots, \frac{\partial u_{\mu}}{\partial x_{i_{1}}}(x)\right), \mu=1, \ldots, m$. For $h: U \rightarrow \mathbb{R}$ we put $h_{+}(x):=$ $\sup (h(x), 0), x \in U$.

Let $\mathscr{V}$ be a real vector space equipped with a norm $|\cdot|$. We say that a function $\Phi: \mathscr{V} \rightarrow \mathbb{R}$ is positively homogeneous of degree $p \in \mathbb{R}$ if $\Phi(t x)=t^{p} \Phi(x)$ for all $t>0$ and $x \in \mathscr{V} \backslash\{0\}$. For $\varepsilon>-1$ we put $|x|^{\varepsilon} x=0$ at $x=0$.

Following Ch. B. Morrey [55], we say that a continuous function $F: \mathbb{R}^{m \times n} \rightarrow \mathbb{R}$ is quasiconvex, if

$$
|B(0,1)| F(\zeta) \leqslant \int_{B(0,1)} F\left(\zeta+\varphi^{\prime}(x)\right) d x
$$

for all $\varphi \in C_{0}^{\infty}\left(B(0,1) ; \mathbb{R}^{m}\right)$ and $\zeta \in \mathbb{R}^{m \times n}$. Let $p \geqslant 1$. Following M. A. Sychev [66], we say that a quasiconvex function $F$ is strictly $p$-quasiconvex if, for $\zeta \in \mathbb{R}^{m \times n}$ and $\varepsilon, C>0$, 
there is $\delta=\delta(\zeta, \varepsilon, C)>0$ such that, for each mapping $\varphi \in C_{0}^{\infty}\left(B(0,1) ; \mathbb{R}^{m}\right)$ satisfying $\left\|\varphi^{\prime}\right\|_{L^{p}\left(B(0,1) ; \mathbb{R}^{m \times n}\right)} \leqslant C|B(0,1)|^{1 / p}$, the condition $\int_{B(0,1)} F\left(\zeta+\varphi^{\prime}(x)\right) d x \leqslant|B(0,1)|(F(\zeta)+\delta)$ implies $\left|\left\{x \in B(0,1):\left|\varphi^{\prime}(x)\right| \geqslant \varepsilon\right\}\right| \leqslant \varepsilon|B(0,1)|$. Observe that in the mathematical literature the term strictly quasiconvexity is also used for another property (which is close but nonequivalent to ours) consisting in the fact that the strict inequality in the definition of quasiconvexity (5) is valid for nonzero mappings $\varphi$ (for example, see [38]). In this article we use the term in the sense of M. A. Sychev's definition [66]. In the case $p>1$ the notion of strictly $p$-quasiconvexity for functions $F$ of this article is equivalent to the notion of strictly closed $p$-quasiconvexity from J. Kristensen's article [44] which is defined in terms of the theory of gradient Young measures (see [44, Proposition 3.4]). Observe that we can replace the ball $B(0,1)$ in the definitions of quasiconvexity and strictly $p$-quasiconvexity by an arbitrary bounded domain $U$ with $|\partial U|=0$ (for example, see [56]). A function $G: \mathbb{R}^{m \times n} \rightarrow \mathbb{R}$ is a null Lagrangian if both functions $G$ and $-G$ are quasiconvex. The term "null Lagrangian" appeared due to the following fact: The Euler-Lagrange equation corresponding to the variational integral $\int_{U} G\left(u^{\prime}(x)\right) d x$ with null Lagrangian $G$ holds identically for all admissible mappings $u: U \subset \mathbb{R}^{n} \rightarrow \mathbb{R}^{m}$ (see [4] and also [5, 36, 56]). The only the affine combinations of minors (called quasiaffine functions) are null Lagrangians [15, 47] (also see $[3,4,5,36,55,56]$ ); i. e.

$$
G(\zeta)=\gamma_{0}+\sum_{k=1}^{\min \{m, n\}} \sum_{J \in \Gamma_{m}^{k}, I \in \Gamma_{n}^{k}} \gamma_{J I} \operatorname{det}_{J I} \zeta, \quad \zeta \in \mathbb{R}^{m \times n},
$$

for some $\gamma_{0}, \gamma_{J I} \in \mathbb{R}$.

\section{Statement of the Main Results}

The principal result on higher integrability of solutions to (1) states as follows

Theorem 2.1 (Higher integrability). Let $n, m, k \in \mathbb{N}$ and $t>k$ such that $2 \leqslant k \leqslant$ $\min \{n, m\}$, and let $V$ be an open set in $\mathbb{R}^{n}$. Suppose that a continuous function $F: \mathbb{R}^{m \times n} \rightarrow \mathbb{R}$ satisfies

$$
F(\zeta) \geqslant c_{F}|\zeta|^{k}, \quad \zeta \in \mathbb{R}^{m \times n}
$$

with some constant $c_{F}>0$, a null Lagrangian $G: \mathbb{R}^{m \times n} \rightarrow \mathbb{R}$ is homogeneous of degree $k$, and a measurable function $H: V \rightarrow \mathbb{R}$ has $H_{+} \in L_{\text {loc }}^{t}(V)$. Then for $K \geqslant 1$ there exist two numbers $\underline{p}=\underline{p}(F, G, K)$ and $\bar{p}=\bar{p}(F, G, K)$ depended only $F, G$, and $K$ with $1 \leqslant \underline{p}<k<\bar{p} \leqslant t$ such that for a given exponent $p>\underline{p}$ every solution $v \in W_{\text {loc }}^{1, p}\left(V ; \mathbb{R}^{m}\right)$ to (1) actually lies in $W_{\text {loc }}^{1, s}\left(V ; \mathbb{R}^{m}\right)$ for all $s \in(\underline{p}, \bar{p})$. Moreover, for a number $\varepsilon>0$, a vector $b \in \mathbb{R}^{m}$, and a test function $\varphi \in C_{0}^{\infty}(V)$ we have the Caccioppoli-type inequality

$$
\left\|\varphi(\cdot) v^{\prime}(\cdot)\right\|_{L^{s}\left(V ; \mathbb{R}^{m \times n}\right)} \leqslant C\left\|\left|(v(\cdot)-b) \otimes \varphi^{\prime}(\cdot)\right|+|\varphi(\cdot)|\left(\varepsilon+\varepsilon^{1-k} H_{+}(\cdot)\right)\right\|_{L^{s}(V)}
$$

with some constant $C=C(F, G, K, s)>0$ depended only $F, G, K$, and $s$.

In the case $H(x) \equiv 0$ a straightforward consequence of Theorem 2.1 is the following.

Corollary 2.2. Under the conditions of Theorem 2.1, there exist two numbers $\underline{p}_{0}=$ $\underline{p}_{0}(F, G, K)$ and $\bar{p}_{0}=\bar{p}_{0}(F, G, K)$ depended only $F, G$, and $K$ with $1 \leqslant \underline{p}_{0}<k<\bar{p}_{0}$ such that for a given exponent $p>\underline{p}_{0}$ every solution $v \in W_{\text {loc }}^{1, p}\left(V ; \mathbb{R}^{m}\right)$ to $(2)$ actually lies in $W_{\text {loc }}^{1, s}\left(V ; \mathbb{R}^{m}\right)$ for all $s \in\left(\underline{p}_{0}, \bar{p}_{0}\right)$. Moreover, for a vector $b \in \mathbb{R}^{m}$ and a test function $\varphi \in C_{0}^{\infty}(V)$ we have the homogeneous Caccioppoli-type inequality

$$
\left\|\varphi(\cdot) v^{\prime}(\cdot)\right\|_{L^{s}\left(V ; \mathbb{R}^{m \times n}\right)} \leqslant C_{0}\left\|(v(\cdot)-b) \otimes \varphi^{\prime}(\cdot)\right\|_{L^{s}\left(V ; \mathbb{R}^{m \times n}\right)}
$$


with some constant $C_{0}=C_{0}(F, G, K, s)>0$ depended only $F, G, K$, and $s$.

Now we apply Theorem 2.1 to improve some results from [19].

Let $n, m, k \in \mathbb{N}$ such that $2 \leqslant k \leqslant \min \{n, m\}$. We need the following hypothesis on continuous functions $F: \mathbb{R}^{m \times n} \rightarrow \mathbb{R}$ and $G: \mathbb{R}^{m \times n} \rightarrow \mathbb{R}$ (see [19]):

(H1) $F$ is quasiconvex;

$\left(\mathrm{H} 1^{\prime}\right) F$ is strictly $k$-quasiconvex;

(H2) $G$ is a null Lagrangian;

(H3) $F$ and $G$ are positively homogeneous of degree $k$;

(H4) $\sup \left\{K \geqslant 0: F(\zeta) \geqslant K G(\zeta), \zeta \in \mathbb{R}^{m \times n}\right\}=1$;

(H5) $c_{F}^{0}:=\inf \left\{F(\zeta): \zeta \in \mathbb{R}^{m \times n},|\zeta|=1\right\}>0$

(H6) $d_{G}:=\sup \left\{\sum_{J \in \Gamma_{m}^{k}, I \in \Gamma_{n}^{k}}\left|\gamma_{J I}\right|\left|x_{I}\right|^{2}: x \in \mathbb{R}^{n},|x|=1\right\}<k c_{F}^{0} /(n-k)$ in the case $k<n$.

Here the coefficients $\gamma_{J I}$ are taken from (6) for the null Lagrangian $G$. By (H3), the representation (6) for the null Lagrangian $G$ consists only of $k \times k$-minors; i. e.,

$$
G(\zeta)=\sum_{J \in \Gamma_{m}^{k}, I \in \Gamma_{n}^{k}} \gamma_{J I} \operatorname{det}_{J I} \zeta, \quad \zeta \in \mathbb{R}^{m \times n} .
$$

Since $F$ is continuous, (H3) implies (7) with $c_{F}=c_{F}^{0}$.

The following theorem on Hölder regularity of solutions to (1) is a straightforward consequence of Theorem 2.1 and [19, Theorem 8].

Theorem 2.3 (Hölder regularity). Suppose $F$ and $G$ satisfy (H2)-(H6). Put $K_{0}=\infty$ for $k=n$ and $K_{0}=\frac{k c_{F}^{0}}{(n-k) d_{G}}$ for $k<n$. Suppose that $K \in\left[1, K_{0}\right)$ and $\delta \in(0,1)$ satisfy the inequality

$$
\frac{K d_{G}}{k c_{F}^{0}} \leqslant \frac{1}{n-k+k \delta}
$$

Let $\underline{p}$ is the exponent from Theorem $2.1, p>\underline{p}$, and $V$ be an open set in $\mathbb{R}^{n}$. Then every solution $v \in W_{\text {loc }}^{1, p}\left(V ; \mathbb{R}^{m}\right)$ to (1) with $H(x) \equiv$ const satisfies the Hölder condition with exponent $\delta$ on each compact subset in $V$.

The next theorem on stability of the class of solutions to (3) is a straightforward consequence of Corollary 2.2 and [19, Theorem 4].

Theorem 2.4 (Stability in the $C$-norme). Suppose that $F$ and $G$ satisfy (H1)-(H6). Let $K \geqslant 1$, and let $\underline{p}_{0}$ denote the exponent from Corollary 2.2. Let $V$ be a domain in $\mathbb{R}^{n}$, and let $U$ be a compact subset in $V$. Then there is a function $\alpha(K)=\alpha_{F, G, V, U}(K)$ defined for $1 \leqslant K<K_{0}$ and such that $\lim _{K \rightarrow 1} \alpha(K)=\alpha(1)=0$ and, for each mapping $v \in W_{\text {loc }}^{1, p}\left(V ; \mathbb{R}^{m}\right)$, $p>\underline{p}_{0}$, which satisfies inequality (2) there is a mapping $u \in W_{\mathrm{loc}}^{1, k}\left(V ; \mathbb{R}^{m}\right)$ which is a solution to $(3)$ such that

$$
\|v-u\|_{C\left(U ; \mathbb{R}^{m}\right)} \leqslant \alpha(K) \operatorname{diam} v(V) .
$$

The following theorem improves Theorem 2.4 in the case when the function $F$ satisfies $\left(\mathrm{H} 1^{\prime}\right)$. In this case, in addition to the estimate (12) of proximity (in the $C$-norm) of solutions of inequality (1) to solutions to equation (3), we obtain proximity estimates (in the $L^{k}$-norm) for the derivatives of these mappings. The theorem is a straightforward consequence of Corollary 2.2 and [19, Theorem 6].

Theorem 2.4 (Stability in the Sobolev norme). Suppose that $F$ and $G$ satisfy (H1') and (H2)-(H6). Then the conclusion of Theorem 2.4 is valid together with (12) and the following inequality:

$$
\left\|v^{\prime}-u^{\prime}\right\|_{L^{k}\left(U ; \mathbb{R}^{m \times n}\right)} \leqslant \alpha(K) \operatorname{diam} v(V) .
$$




\section{Auxiliary Lemmas}

In the proof of the higher integrability theorem we need the following lemmas.

Lemma 3.1. Let $n, m, k \in \mathbb{N}, 2 \leqslant k \leqslant \min (m, n), p \geqslant 1, b \in \mathbb{R}^{m}, I=\left(i_{1}, \ldots, i_{k}\right) \in \Gamma_{n}^{k}$, $J=\left(j_{1}, \ldots, j_{k}\right) \in \Gamma_{m}^{k}$, and $V \subset \mathbb{R}^{n}$ be an open set. For a mapping $v: V \rightarrow \mathbb{R}^{m}$ and a function $\varphi: V \rightarrow \mathbb{R}$ define the mappings $w=\left(w_{1}, \ldots, w_{m}\right): V \rightarrow \mathbb{R}^{m}$ and $h=\left(h_{1}, \ldots, h_{m}\right): V \rightarrow \mathbb{R}^{m}$ by the rules $w(\cdot):=v(\cdot)-b$ and $h(\cdot):=\varphi(\cdot) w(\cdot)$. If $v$ and $\varphi$ are differentiable at $x \in V$, then the following inequality

$$
\begin{aligned}
\left.|| \varphi(x) v^{\prime}(x)\right|^{p-k} \operatorname{det}_{J I}\left(\varphi(x) v^{\prime}(x)\right) & -\left|h^{\prime}(x)\right|^{p-k} \operatorname{det}_{J I} h^{\prime}(x) \mid \\
& \leqslant(2 k+p)\left|w(x) \otimes \varphi^{\prime}(x)\right|\left(\left|h^{\prime}(x)\right|+\left|w(x) \otimes \varphi^{\prime}(x)\right|\right)^{p-1}
\end{aligned}
$$

holds.

$\triangleleft$ Observe that

$$
\varphi(x) v^{\prime}(x)=h^{\prime}(x)-w(x) \otimes \varphi^{\prime}(x) .
$$

We have

$$
\operatorname{det}_{J I}\left(h^{\prime}-w \otimes \varphi^{\prime}\right)=\operatorname{det}\left(\begin{array}{c}
\partial_{I} h_{j_{1}}-w_{j_{1}} \partial_{I} \varphi \\
\vdots \\
\partial_{I} h_{j_{k}}-w_{j_{k}} \partial_{I} \varphi
\end{array}\right) .
$$

Hence, $\operatorname{det}_{J I}\left(h^{\prime}-w \otimes \varphi^{\prime}\right)=\operatorname{det}_{J I} h^{\prime}-\sum_{\varkappa=1}^{k} A_{\varkappa}$ where

$$
A_{\varkappa}:=\operatorname{det}\left(\begin{array}{c}
\partial_{I} h_{j_{1}} \\
\vdots \\
\partial_{I} h_{j_{\varkappa-1}} \\
w_{j_{\varkappa}} \partial_{I} \varphi \\
\partial_{I} h_{j_{\varkappa+1}}-w_{j_{\varkappa+1}} \partial_{I} \varphi \\
\vdots \\
\partial_{I} h_{j_{k}}-w_{j_{k}} \partial_{I} \varphi
\end{array}\right) .
$$

Observe that

$$
\begin{aligned}
\left|A_{\varkappa}\right| \leqslant & \left|\partial_{I} h_{j_{1}}\right| \ldots\left|\partial_{I} h_{j_{\varkappa-1}}\right|\left|w_{j_{\varkappa}} \partial_{I} \varphi\right|\left|\partial_{I} h_{j_{\varkappa+1}}-w_{j_{\varkappa+1}} \partial_{I} \varphi\right| \ldots\left|\partial_{I} h_{j_{k}}-w_{j_{k}} \partial_{I} \varphi\right| \\
& \leqslant\left|w_{J} \otimes \partial_{I} \varphi\right|\left|\partial_{I} h_{J}\right|^{\varkappa-1}\left|\partial_{I} h_{J}-w_{J} \otimes \partial_{I} \varphi\right|^{k-\varkappa} \leqslant\left|w \otimes \varphi^{\prime}\right|\left|h^{\prime}\right|^{\varkappa-1}\left|h^{\prime}-w \otimes \varphi^{\prime}\right|^{k-\varkappa} .
\end{aligned}
$$

Therefore,

$$
\left|\operatorname{det}_{J I}\left(h^{\prime}-\varphi v^{\prime}\right)-\operatorname{det}_{J I} h^{\prime}\right| \leqslant\left|w \otimes \varphi^{\prime}\right| \sum_{\varkappa=1}^{k}\left|h^{\prime}\right|^{\varkappa-1}\left|h^{\prime}-w \otimes \varphi^{\prime}\right|^{k-\varkappa} .
$$

Let us consider 2 cases.

Case 1:

$$
0<\left|h^{\prime}(x)-w(x) \otimes \varphi^{\prime}(x)\right| \leqslant\left|h^{\prime}(x)\right|
$$

We have

$$
\begin{aligned}
& || h^{\prime}-\left.w \otimes \varphi^{\prime}\right|^{p-k} \operatorname{det}_{J I}\left(h^{\prime}-w \otimes \varphi^{\prime}\right)-\left|h^{\prime}\right|^{p-k} \operatorname{det}_{J I} h^{\prime} \mid \\
& \leqslant\left|h^{\prime}-w \otimes \varphi^{\prime}\right|^{p}\left|\operatorname{det}_{J I}\left(h^{\prime}-w \otimes \varphi^{\prime}\right)\right||| h^{\prime}-\left.w \otimes \varphi^{\prime}\right|^{-k}-\left|h^{\prime}\right|^{-k} \mid \\
& +\left|h^{\prime}-w \otimes \varphi^{\prime}\right|^{p}\left|h^{\prime}\right|^{-k}\left|\operatorname{det}_{J I}\left(h^{\prime}-w \otimes \varphi^{\prime}\right)-\operatorname{det}_{J I} h^{\prime}\right| \\
& +\left|h^{\prime}\right|^{-k}\left|\operatorname{det}_{J I} h^{\prime}\right||| h^{\prime}-\left.w \otimes \varphi^{\prime}\right|^{p}-\left|h^{\prime}\right|^{p} \mid .
\end{aligned}
$$


We estimate each term on the right-hand side of (18). We obviously have the inequalities

$$
a_{1}^{-k}-a_{2}^{-k} \leqslant k\left(a_{2}-a_{1}\right) a_{1}^{-k-1}
$$

and

$$
a_{2}^{p}-a_{1}^{p} \leqslant p\left(a_{2}-a_{1}\right) a_{2}^{p-1}
$$

for $0<a_{1} \leqslant a_{2}$. Using (17) and (19), we get

$$
\begin{aligned}
&|| h^{\prime}-\left.w \otimes \varphi^{\prime}\right|^{-k}-\left|h^{\prime}\right|^{-k} \mid=\left|h^{\prime}-w \otimes \varphi^{\prime}\right|^{-k}-\left|h^{\prime}\right|^{-k} \\
& \leqslant k\left(\left|h^{\prime}\right|-\left|h^{\prime}-w \otimes \varphi^{\prime}\right|\right)\left|h^{\prime}-w \otimes \varphi^{\prime}\right|^{-k-1} \leqslant k\left|w \otimes \varphi^{\prime}\right|\left|h^{\prime}-w \otimes \varphi^{\prime}\right|^{-k-1} .
\end{aligned}
$$

By Hadamard's inequality

$$
\left|\operatorname{det}_{J I}\left(h^{\prime}-w \otimes \varphi^{\prime}\right)\right| \leqslant\left|h^{\prime}-w \otimes \varphi^{\prime}\right|^{k}
$$

Combining (21) with (22), we have

$$
\begin{aligned}
& \left.\left|h^{\prime}-w \otimes \varphi^{\prime}\right|\right|^{p}\left|\operatorname{det}_{J I}\left(h^{\prime}-w \otimes \varphi^{\prime}\right)\right||| h^{\prime}-\left.w \otimes \varphi^{\prime}\right|^{-k}-\left|h^{\prime}\right|^{-k} \mid \\
& \leqslant k\left|w \otimes \varphi^{\prime}\right|\left|h^{\prime}-w \otimes \varphi^{\prime}\right|^{p-1} \leqslant k\left|w \otimes \varphi^{\prime}\right|\left(\left|h^{\prime}\right|+\left|w \otimes \varphi^{\prime}\right|\right)^{p-1} .
\end{aligned}
$$

Using (16) and (17), we obtain

$$
\begin{aligned}
& \left|h^{\prime}-w \otimes \varphi^{\prime}\right|^{p}\left|h^{\prime}\right|^{-k}\left|\operatorname{det}_{J I}\left(h^{\prime}-w \otimes \varphi^{\prime}\right)-\operatorname{det}_{J I} h^{\prime}\right| \\
& \leqslant\left|h^{\prime}-w \otimes \varphi^{\prime}\right|^{p}\left|h^{\prime}\right|^{-k}\left|w \otimes \varphi^{\prime}\right| \sum_{\varkappa=1}^{k}\left|h^{\prime}\right|^{\varkappa-1}\left|h^{\prime}-w \otimes \varphi^{\prime}\right|^{k-\varkappa} \\
& \leqslant\left|w \otimes \varphi^{\prime}\right|\left|h^{\prime}-w \otimes \varphi^{\prime}\right|^{p-1} \sum_{\varkappa=1}^{k}\left(\left|h^{\prime}-w \otimes \varphi^{\prime}\right| /\left|h^{\prime}\right|\right)^{k-\varkappa+1} \\
& \leqslant k\left|w \otimes \varphi^{\prime}\right|\left|h^{\prime}-w \otimes \varphi^{\prime}\right|^{p-1} \leqslant k\left|w \otimes \varphi^{\prime}\right|\left(\left|h^{\prime}\right|+\left|w \otimes \varphi^{\prime}\right|\right)^{p-1} .
\end{aligned}
$$

Using (17) and (20), we get

$$
\begin{aligned}
|| h^{\prime}-\left.w \otimes \varphi^{\prime}\right|^{p}-\left.\left|h^{\prime}\right|^{p}|=| h^{\prime}\right|^{p}-\left|h^{\prime}-w \otimes \varphi^{\prime}\right|^{p} & \\
& \leqslant p\left(\left|h^{\prime}\right|-\left|h^{\prime}-w \otimes \varphi^{\prime}\right|\right)\left|h^{\prime}\right|^{p-1} \leqslant p\left|w \otimes \varphi^{\prime}\right|\left|h^{\prime}\right|^{p-1} .
\end{aligned}
$$

By Hadamard's inequality

$$
\left|\operatorname{det}_{J I} h^{\prime}\right| \leqslant\left|h^{\prime}\right|^{k}
$$

Combining (25) with (26), we have

$$
\begin{aligned}
\left|h^{\prime}\right|^{-k}\left|\operatorname{det}_{J I} h^{\prime}\right||| h^{\prime}-\left.w \otimes \varphi^{\prime}\right|^{p}-\left.\left|h^{\prime}\right|^{p}|\leqslant p| w \otimes \varphi^{\prime}|| h^{\prime}\right|^{p-1} & \\
& \leqslant p\left|w \otimes \varphi^{\prime}\right|\left(\left|h^{\prime}\right|+\left|w \otimes \varphi^{\prime}\right|\right)^{p-1} .
\end{aligned}
$$

Using (15), (18), (23), (24), and (27), we obtain (14) in case 1.

Case 2:

$$
0<\left|h^{\prime}(x)\right| \leqslant\left|h^{\prime}(x)-w(x) \otimes \varphi^{\prime}(x)\right| .
$$


We have

$$
\begin{aligned}
& || h^{\prime}-\left.w \otimes \varphi^{\prime}\right|^{p-k} \operatorname{det}_{J I}\left(h^{\prime}-w \otimes \varphi^{\prime}\right)-\left|h^{\prime}\right|^{p-k} \operatorname{det}_{J I} h^{\prime} \mid \\
& \leqslant\left|h^{\prime}-w \otimes \varphi^{\prime}\right|^{-k}\left|\operatorname{det}_{J I}\left(h^{\prime}-w \otimes \varphi^{\prime}\right)\right||| h^{\prime}-\left.w \otimes \varphi^{\prime}\right|^{p}-\left|h^{\prime}\right|^{p} \mid \\
& +\left|h^{\prime}-w \otimes \varphi^{\prime}\right|^{-k}\left|h^{\prime}\right|^{p}\left|\operatorname{det}_{J I}\left(h^{\prime}-w \otimes \varphi^{\prime}\right)-\operatorname{det}_{J I} h^{\prime}\right| \\
& \quad+\left|h^{\prime}\right|^{p}\left|\operatorname{det}_{J I} h^{\prime}\right||| h^{\prime}-\left.w \otimes \varphi^{\prime}\right|^{-k}-\left|h^{\prime}\right|^{-k} \mid .
\end{aligned}
$$

We estimate each term on the right-hand side of (29). Using (20) and (28), we get

$$
\begin{aligned}
& || h^{\prime}-\left.w \otimes \varphi^{\prime}\right|^{p}-\left|h^{\prime}\right|^{p}|=| h^{\prime}-\left.w \otimes \varphi^{\prime}\right|^{p}-\left|h^{\prime}\right|^{p} \\
& \leqslant p\left(\left|h^{\prime}-w \otimes \varphi^{\prime}\right|-\left|h^{\prime}\right|\right)\left|h^{\prime}-w \otimes \varphi^{\prime}\right|^{p-1} \leqslant p\left|w \otimes \varphi^{\prime}\right|\left|h^{\prime}-w \otimes \varphi^{\prime}\right|^{p-1} .
\end{aligned}
$$

If we combain this with (22), we obtain

$$
\begin{aligned}
\left|h^{\prime}-w \otimes \varphi^{\prime}\right|^{-k} \mid \operatorname{det}_{J I}\left(h^{\prime}\right. & \left.-w \otimes \varphi^{\prime}\right)||\left|h^{\prime}-w \otimes \varphi^{\prime}\right|^{p}-\left|h^{\prime}\right|^{p} \mid \\
& \leqslant p\left|w \otimes \varphi^{\prime}\right|\left|h^{\prime}-w \otimes \varphi^{\prime}\right|^{p-1} \leqslant p\left|w \otimes \varphi^{\prime}\right|\left(\left|h^{\prime}\right|+\left|w \otimes \varphi^{\prime}\right|\right)^{p-1} .
\end{aligned}
$$

Using (16) and (28), we get

$$
\begin{aligned}
& \left|h^{\prime}-w \otimes \varphi^{\prime}\right|^{-k}\left|h^{\prime}\right|^{p}\left|\operatorname{det}_{J I}\left(h^{\prime}-w \otimes \varphi^{\prime}\right)-\operatorname{det}_{J I} h^{\prime}\right| \\
& \leqslant\left|h^{\prime}-w \otimes \varphi^{\prime}\right|^{-k}\left|h^{\prime}\right|^{p}\left|w \otimes \varphi^{\prime}\right| \sum_{\varkappa=1}^{k}\left|h^{\prime}\right|^{\varkappa-1}\left|h^{\prime}-w \otimes \varphi^{\prime}\right|^{k-\varkappa} \\
& \leqslant\left|w \otimes \varphi^{\prime}\right|\left|h^{\prime}\right|^{p-1} \sum_{\varkappa=1}^{k}\left(\left|h^{\prime}\right| /\left|h^{\prime}-w \otimes \varphi^{\prime}\right|\right)^{\varkappa} \\
& \leqslant k\left|w \otimes \varphi^{\prime}\right|\left|h^{\prime}\right|^{p-1} \leqslant k\left|w \otimes \varphi^{\prime}\right|\left(\left|h^{\prime}\right|+\left|w \otimes \varphi^{\prime}\right|\right)^{p-1} .
\end{aligned}
$$

Using (19) and (28), we have

$$
\begin{aligned}
|| h^{\prime}-\left.w \otimes \varphi^{\prime}\right|^{-k}-\left.\left|h^{\prime}\right|^{-k}|=| h^{\prime}\right|^{-k}-\left|h^{\prime}-w \otimes \varphi^{\prime}\right|^{-k} & \\
& \leqslant k\left(\left|h^{\prime}-w \otimes \varphi^{\prime}\right|-\left|h^{\prime}\right|\right)\left|h^{\prime}\right|^{-k-1} \leqslant k\left|w \otimes \varphi^{\prime}\right|\left|h^{\prime}\right|^{-k-1} .
\end{aligned}
$$

If we combain this with (26), we obtain

$$
\begin{aligned}
\left|h^{\prime}\right|^{p}\left|\operatorname{det}_{J I} h^{\prime}\right|\left|h^{\prime}-w \otimes \varphi^{\prime}\right|^{-k}-\left.\left|h^{\prime}\right|^{-k}|\leqslant k| w \otimes \varphi^{\prime}|| h^{\prime}\right|^{p-1} & \\
& \leqslant k\left|w \otimes \varphi^{\prime}\right|\left(\left|h^{\prime}\right|+\left|w \otimes \varphi^{\prime}\right|\right)^{p-1} .
\end{aligned}
$$

Combining (15) and (29)-(32), we get (14) in case 2. $\triangleright$

Lemma 3.2. Suppose that a null Lagrangian $G$ is homogeneous of degree $k$. Under the conditions of Lemma 3.1, we have

$$
\begin{aligned}
& \|\left.\varphi(x) v^{\prime}(x)\right|^{p-k} G\left(\varphi(x) v^{\prime}(x)\right)-\left|h^{\prime}(x)\right|^{p-k} G\left(h^{\prime}(x)\right) \mid \\
& \leqslant C\left|w(x) \otimes \varphi^{\prime}(x)\right|\left(\left|h^{\prime}(x)\right|+\left|w(x) \otimes \varphi^{\prime}(x)\right|\right)^{p-1}
\end{aligned}
$$

with some constant $C=C(G, p)$ depended only on $G$ and $p$. 
$\triangleleft$ Using (10) and (14), we get

$$
\begin{aligned}
& \|\left.\varphi(x) v^{\prime}(x)\right|^{p-k} G\left(\varphi(x) v^{\prime}(x)\right)-\left|h^{\prime}(x)\right|^{p-k} G\left(h^{\prime}(x)\right) \mid \\
& \leqslant\left.\sum_{J \in \Gamma_{m}^{k}, I \in \Gamma_{n}^{k}}\left|\gamma_{J I}\right||| \varphi(x) v^{\prime}(x)\right|^{p-k} \operatorname{det}_{J I}\left(\varphi(x) v^{\prime}(x)\right)-\left|h^{\prime}(x)\right|^{p-k} \operatorname{det}_{J I} h^{\prime}(x) \mid \\
& \quad \leqslant(2 k+p)\left(\sum_{J \in \Gamma_{m}^{k}, I \in \Gamma_{n}^{k}}\left|\gamma_{J I}\right|\right)\left|w(x) \otimes \varphi^{\prime}(x)\right|\left(\left|h^{\prime}(x)\right|+\left|w(x) \otimes \varphi^{\prime}(x)\right|\right)^{p-1} . \triangleright
\end{aligned}
$$

REMARK 3.3. We can use $C(G, p)=(2 k+p) \sum_{J \in \Gamma_{m}^{k}, I \in \Gamma_{n}^{k}}\left|\gamma_{J I}\right|$ as the constant in (33). Here $\gamma_{J I}$ are the coefficients from (10).

Also, we need the following version of Gehring's lemma (for example, see [36, Corollary 14.3.1]):

Lemma 3.4. Suppose $f$ and $g$ are non-negative functions of the class $L^{q}\left(\mathbb{R}^{n}\right), 1<q<\infty$, and satisfy

$$
\left(\frac{1}{|B(a, R)|} \int_{B(a, R)} f^{q}\right)^{1 / q} \leqslant \frac{A}{|B(a, 2 R)|} \int_{B(a, 2 R)} f+\left(\frac{1}{|B(a, 2 R)|} \int_{B(a, 2 R)} g^{q}\right)^{1 / q}
$$

for all balls $B(a, R) \subset \mathbb{R}^{n}$ and some constant $A>0$. Then the inequality

$$
\int f^{q^{\prime}} \leqslant C \int g^{q^{\prime}}
$$

holds with some exponent $q^{\prime}=q^{\prime}(n, q, A)>q$ and some constant $C=C(n, q, A)>0$ depended only $n, q$, and $A$.

\section{Proof of the Higher Intagrability Theorem}

We are now in a position to prove the higher intagrability theorem.

$\triangleleft$ Proof of Theorem 2.1. Let $V \subset \mathbb{R}^{n}$ be an open set and $1<p \leqslant t$. A suitable range of Sobolev exponents $p$ will be defined below (see (46), (47), and (48)).

Fix a solution $v \in W_{\text {loc }}^{1, p}\left(V ; \mathbb{R}^{m}\right)$ to $(1)$. Consider $\varphi \in C_{0}^{\infty}(V)$. We may assume that $\varphi$ is non-negative as otherwise we could consider $|\varphi|$ which has not effect on the required Caccioppoli-type inequality (8). Using (1) and $F(\zeta) \geqslant c_{F}|\zeta|^{k}$, we get

$$
\left|v^{\prime}(x)\right|^{k} \leqslant c_{F}^{-1} K G\left(v^{\prime}(x)\right)+c_{F}^{-1} H_{+}(x) \quad \text { a.e. } V \text {. }
$$

Multipling both sides by $\varphi^{p}(x)\left|v^{\prime}(x)\right|^{p-k}$ and using $k$-homogeneity of $G$, we obtain

$$
\begin{aligned}
\left|\varphi(x) v^{\prime}(x)\right|^{p} \leqslant c_{F}^{-1} K\left|\varphi(x) v^{\prime}(x)\right|^{p-k} G\left(\varphi(x) v^{\prime}(x)\right) & \\
& +c_{F}^{-1} \varphi^{p}(x)\left|v^{\prime}(x)\right|^{p-k} H_{+}(x) \quad \text { a. e. } \quad V .
\end{aligned}
$$

Let $\varepsilon>0$. Put $V_{1}:=\left\{x \in V:\left|v^{\prime}(x)\right| \geqslant \varepsilon\right\}$ and $V_{2}:=\left\{x \in V:\left|v^{\prime}(x)\right|<\varepsilon\right\}$. We have $V_{1} \cap V_{2}=\varnothing$ and $\left|V \backslash\left(V_{1} \cup V_{2}\right)\right|=0$. Then

$$
\int_{V}\left|\varphi v^{\prime}\right|^{p}=\int_{V_{1}}\left|\varphi v^{\prime}\right|^{p}+\int_{V_{2}}\left|\varphi v^{\prime}\right|^{p}
$$


We estimate each term on the right-hand side of (36).

Using (35), we have

$$
\int_{V_{1}}\left|\varphi v^{\prime}\right|^{p} \leqslant c_{F}^{-1} K \int_{V_{1}}\left|\varphi v^{\prime}\right|^{p-k} G\left(\varphi v^{\prime}\right)+c_{F}^{-1} \int_{V_{1}} \varphi^{p}\left|v^{\prime}\right|^{p-k} H_{+} .
$$

We estimate each term on the right-hand side of (37).

Using (10) and Hadamard's inequality, we have $-G(\zeta) \leqslant|G(\zeta)| \leqslant C_{1}(G)|\zeta|^{k}, \zeta \in \mathbb{R}^{m \times n}$, with $C_{1}(G):=\sum_{J \in \Gamma_{m}^{k}, I \in \Gamma_{n}^{k}}\left|\gamma_{J I}\right|$. Here $\gamma_{J I}$ are the coefficients from (10). Then $|\zeta|^{p-k} G(\zeta)+$ $C_{1}(G)|\zeta|^{p} \geqslant 0$. Therefore,

$$
\int_{V_{2}}\left|\varphi v^{\prime}\right|^{p-k} G\left(\varphi v^{\prime}\right)+C_{1}(G) \int_{V_{2}}\left|\varphi v^{\prime}\right|^{p} \geqslant 0 .
$$

Consequently,

$$
\begin{aligned}
\int_{V_{1}}\left|\varphi v^{\prime}\right|^{p-k} G\left(\varphi v^{\prime}\right) \leqslant \int_{V_{1}}\left|\varphi v^{\prime}\right|^{p-k} G\left(\varphi v^{\prime}\right)+\int_{V_{2}}\left|\varphi v^{\prime}\right|^{p-k} G\left(\varphi v^{\prime}\right) & \\
& +C_{1}(G) \int_{V_{2}}\left|\varphi v^{\prime}\right|^{p}=\int_{V}\left|\varphi v^{\prime}\right|^{p-k} G\left(\varphi v^{\prime}\right)+C_{1}(G) \int_{V_{2}}\left|\varphi v^{\prime}\right|^{p} .
\end{aligned}
$$

We estimate each term on the right-hand side of (38).

Let $b \in \mathbb{R}^{m}$. Define the auxiliary mappings $w: V \rightarrow \mathbb{R}^{m}$ and $h: \mathbb{R}^{n} \rightarrow \mathbb{R}^{m}$ by the rules $w(x):=v(x)-b, x \in V$, and

$$
h(x):= \begin{cases}\varphi(x) w(x), & x \in V, \\ 0, & x \in \mathbb{R}^{n} \backslash V .\end{cases}
$$

Then $h \in W^{1, p}\left(\mathbb{R}^{n} ; \mathbb{R}^{m}\right)$. We have $\varphi v^{\prime}=h^{\prime}-w \otimes \varphi^{\prime}$.

Successively using Lemma 3.2, (10), and [22, Theorem 2.1], we deduce

$$
\begin{aligned}
\int_{V}\left|\varphi v^{\prime}\right|^{p-k} G\left(\varphi v^{\prime}\right) & \leqslant \int\left|h^{\prime}\right|^{p-k} G\left(h^{\prime}\right)+C(G, p) \int_{V}\left(\left|h^{\prime}\right|+\left|w \otimes \varphi^{\prime}\right|\right)^{p-1}\left|w \otimes \varphi^{\prime}\right| \\
= & \sum_{J \in \Gamma_{m}^{k}, I \in \Gamma_{n}^{k}} \gamma_{J I} \int\left|h^{\prime}\right|^{p-k} \operatorname{det}_{J I}\left(h^{\prime}\right)+C(G, p) \int_{V}\left(\left|h^{\prime}\right|+\left|w \otimes \varphi^{\prime}\right|\right)^{p-1}\left|w \otimes \varphi^{\prime}\right| \\
& \leqslant C_{2}(G)|1-p / k| \int\left|h^{\prime}\right|^{p}+C(G, p) \int_{V}\left(\left|h^{\prime}\right|+\left|w \otimes \varphi^{\prime}\right|\right)^{p-1}\left|w \otimes \varphi^{\prime}\right|
\end{aligned}
$$

where $C_{2}(G):=C(k) C_{1}(G), C(k)$ is from [22, Theorem 2.1], and $C(G, p)=(2 k+p) C_{1}(G)$ is from Remark 3.3.

Since $\left|v^{\prime}\right|<\varepsilon$ on $V_{2}$, we have

$$
\int_{V_{2}}\left|\varphi v^{\prime}\right|^{p} \leqslant \varepsilon \int_{V_{2}}\left|\varphi v^{\prime}\right|^{p-1} \varphi
$$


Combining (38), (39), and (40), we get

$$
\begin{aligned}
\int_{V_{1}}\left|\varphi v^{\prime}\right|^{p-k} G\left(\varphi v^{\prime}\right) \leqslant & C_{2}(G)|1-p / k| \int\left|h^{\prime}\right|^{p} \\
& +C(G, p) \int_{V}\left(\left|h^{\prime}\right|+\left|w \otimes \varphi^{\prime}\right|\right)^{p-1}\left|w \otimes \varphi^{\prime}\right|+\varepsilon C_{1}(G) \int_{V_{2}}\left|\varphi v^{\prime}\right|^{p-1} \varphi .
\end{aligned}
$$

Since $\left|v^{\prime}\right| \geqslant \varepsilon$ on $V_{1}$, we have

$$
\int_{V_{1}} \varphi^{p}\left|v^{\prime}\right|^{p-k} H_{+} \leqslant \varepsilon^{1-k} \int_{V_{1}}\left|\varphi v^{\prime}\right|^{p-1} \varphi H_{+} .
$$

Using (37), (41), and (42), we obtain

$$
\begin{aligned}
& \int_{V_{1}}\left|\varphi v^{\prime}\right|^{p} \leqslant c_{F}^{-1} K C_{2}(G)|1-p / k| \int\left|h^{\prime}\right|^{p} \\
& +c_{F}^{-1} K C(G, p) \int_{V}\left(\left|h^{\prime}\right|+\left|w \otimes \varphi^{\prime}\right|\right)^{p-1}\left|w \otimes \varphi^{\prime}\right| \\
& +\varepsilon c_{F}^{-1} K C_{1}(G) \int_{V_{2}}\left|\varphi v^{\prime}\right|^{p-1} \varphi+\varepsilon^{1-k} c_{F}^{-1} \int_{V_{1}}\left|\varphi v^{\prime}\right|^{p-1} \varphi H_{+} .
\end{aligned}
$$

Now combining (36), (40), and (43), and using $\left|\varphi v^{\prime}\right| \leqslant\left|h^{\prime}\right|+\left|w \otimes \varphi^{\prime}\right|$, we get

$$
\begin{gathered}
\int_{V}\left|\varphi v^{\prime}\right|^{p} \leqslant c_{F}^{-1} K C_{2}(G)|1-p / k| \int\left|h^{\prime}\right|^{p} \\
+c_{F}^{-1} K C(G, p) \int_{V}\left(\left|h^{\prime}\right|+\left|w \otimes \varphi^{\prime}\right|\right)^{p-1}\left|w \otimes \varphi^{\prime}\right| \\
+\varepsilon\left(c_{F}^{-1} K C_{1}(G)+1\right) \int_{V_{2}}\left|\varphi v^{\prime}\right|^{p-1} \varphi+\varepsilon^{1-k} c_{F}^{-1} \int_{V_{1}}\left|\varphi v^{\prime}\right|^{p-1} \varphi H_{+} \\
\leqslant c_{F}^{-1} K C_{2}(G)|1-p / k| \int_{\int^{\prime}} \mid h^{\prime}+\left[c_{F}^{-1} K\left(C(G, p)+C_{1}(G)+1\right)+1\right] \\
\times \int_{V}\left(\left|h^{\prime}\right|+\left|w \otimes \varphi^{\prime}\right|\right)^{p-1}\left(\left|w \otimes \varphi^{\prime}\right|+\varphi\left(\varepsilon+\varepsilon^{1-k} H_{+}\right)\right) .
\end{gathered}
$$

Observe that $\left|h^{\prime}\right|^{p} \leqslant 2^{p-1}\left(\left|\varphi v^{\prime}\right|^{p}+\left|w \otimes \varphi^{\prime}\right|^{p}\right)$. By (44), we get

$$
\begin{aligned}
\int\left|h^{\prime}\right|^{p} \leqslant 2^{p-1} \int_{V}\left|\varphi v^{\prime}\right|^{p}+2^{p-1} \int_{V}\left|w \otimes \varphi^{\prime}\right|^{p} \leqslant 2^{p-1} c_{F}^{-1} K C_{2}(G)|1-p / k| \int\left|h^{\prime}\right|^{p} \\
+2^{p-1}\left[c_{F}^{-1} K\left(C(G, p)+C_{1}(G)+1\right)+2\right] \\
\quad \times \int_{V}\left(\left|h^{\prime}\right|+\left|w \otimes \varphi^{\prime}\right|\right)^{p-1}\left(\left|w \otimes \varphi^{\prime}\right|+\varphi\left(\varepsilon+\varepsilon^{1-k} H_{+}\right)\right) .
\end{aligned}
$$


Put

$$
\underline{p}=\underline{p}(F, G, K):=\inf \left\{p \geqslant 1: 2^{p-1} c_{F}^{-1} K C_{2}(G)|1-p / k|<1\right\}
$$

and

$$
\bar{p}=\bar{p}(F, G, K, t):=\sup \left\{p \leqslant t: 2^{p-1} c_{F}^{-1} K C_{2}(G)|1-p / k|<1\right\} .
$$

For

$$
p \in(\underline{p}, \bar{p})
$$

we have $2^{p-1} c_{F}^{-1} K C_{2}(G)|1-p / k|<1$. From (45) we derive

$$
\int\left|h^{\prime}\right|^{p} \leqslant C_{3}(F, G, K, p) \int_{V}\left(\left|h^{\prime}\right|+\left|w \otimes \varphi^{\prime}\right|\right)^{p-1}\left(\left|w \otimes \varphi^{\prime}\right|+\varphi\left(\varepsilon+\varepsilon^{1-k} H_{+}\right)\right),
$$

where $C_{3}(F, G, K, p):=\frac{2^{p-1}\left[c_{F}^{-1} K\left(C(G, p)+C_{1}(G)+1\right)+2\right]}{1-2^{p-1} c_{F}^{-1} K C_{2}(G)|1-p / k|}$.

Successively using $\left(\left|h^{\prime}\right|+\left|w \otimes \varphi^{\prime}\right|\right)^{p} \leqslant 2^{p-1}\left(\left|h^{\prime}\right|^{p}+\left|w \otimes \varphi^{\prime}\right|^{p}\right)$, (49), and Hölder's inequality, we obtain

$$
\begin{aligned}
& \int_{V}\left(\left|h^{\prime}\right|+\left|w \otimes \varphi^{\prime}\right|\right)^{p} \leqslant 2^{p-1}\left(\int\left|h^{\prime}\right|^{p}+\int_{V}\left|w \otimes \varphi^{\prime}\right|^{p}\right) \\
& \leqslant C(F, G, K, p) \int_{V}\left(\left|h^{\prime}\right|+\left|w \otimes \varphi^{\prime}\right|\right)^{p-1}\left(\left|w \otimes \varphi^{\prime}\right|+\varphi\left(\varepsilon+\varepsilon^{1-k} H_{+}\right)\right) \\
& \leqslant C(F, G, K, p)\left(\int_{V}\left(\left|h^{\prime}\right|+\left|w \otimes \varphi^{\prime}\right|\right)^{p}\right)^{\frac{1}{p-1}}\left(\int_{V}\left(\left|w \otimes \varphi^{\prime}\right|+\varphi\left(\varepsilon+\varepsilon^{1-k} H_{+}\right)\right)^{p}\right)^{\frac{1}{p}}
\end{aligned}
$$

where $C(F, G, K, p):=2^{p-1}\left(C_{3}(F, G, K, p)+1\right)$. Therefore,

$$
\left\|\left|h^{\prime}\right|+\left|w \otimes \varphi^{\prime}\right|\right\|_{L^{p}(V)} \leqslant C(F, G, K, p)\left\|\left|w \otimes \varphi^{\prime}\right|+\varphi\left(\varepsilon+\varepsilon^{1-k} H_{+}\right)\right\|_{L^{p}(V)} .
$$

Using $\left|\varphi v^{\prime}\right| \leqslant\left|h^{\prime}\right|+\left|w \otimes \varphi^{\prime}\right|$ and $w=v-b$, we get the Caccioppoli-type estimate

$$
\left\|\varphi v^{\prime}\right\|_{L^{p}\left(V ; \mathbb{R}^{m \times n}\right)} \leqslant C(F, G, K, p)\left\|\left|(v-b) \otimes \varphi^{\prime}\right|+\varphi\left(\varepsilon+\varepsilon^{1-k} H_{+}\right)\right\|_{L^{p}(V)} .
$$

Of course now we observe that this inequality holds with $p$ replaced by $s$ for any $s \in(\underline{p}, \bar{p})$, provided we know a priori that $v \in W_{\text {loc }}^{1, s}\left(V ; \mathbb{R}^{m}\right)$.

Let $S=\left\{s \in(\underline{p}, \bar{p}): v \in W_{\text {loc }}^{1, s}\left(V ; \mathbb{R}^{m}\right)\right\}$. We have $p \in S$. Therefore, $S \neq \varnothing$. For $s \in S$ we have (8); the constant $C=C(F, G, K, s)$ which depends continuously on $s$ is finite in the range $(\underline{p}, \bar{p})$ but may blow up at the endpoints. If we combine this with the Sobolev embedding theorem, we obtain that $S$ is relatively closed in $(p, \bar{p})$. The theorem will be proved if we can show that $S$ is open. Obviously, if $s \in S$, then $(\underline{p}, \bar{s}] \subset(\underline{p}, \bar{p})$. We are therefore left only with the task of showing higher integrability of the differential. It is at this point that Gehring's lemma comes to the rescue. Using (50), we easily derive reverse Hölder inequalities for $v^{\prime}$.

Consider $x_{0} \in V$ and $0<R<\operatorname{dist}\left(x_{0}, V\right) / 3$. Put $B_{R}:=B\left(x_{0}, R\right)$ and $B_{2 R}:=B\left(x_{0}, 2 R\right)$. Let $\eta \in C_{0}^{\infty}\left(B_{2 R}\right)$ be a nonnegative function such that

$$
\eta=1 \text { on } B_{R}, \quad 0 \leqslant \eta \leqslant 1 \text { and }\left|\eta^{\prime}\right| \leqslant \frac{C(n)}{R} \text { on } B_{2 R} .
$$


Substituting $\varphi$ by $\eta$ and $b$ by $v_{B_{2 R}}:=\frac{1}{\left|B_{2 R}\right|} \int_{B_{2 R}} v$ in (50), we get

$$
\left\|\eta v^{\prime}\right\|_{L^{p}\left(B_{2 R} ; \mathbb{R}^{m \times n}\right)} \leqslant C(F, G, K, p)\left\|\left|\left(v-v_{B_{2 R}}\right) \otimes \eta^{\prime}\right|+\eta\left(\varepsilon+\varepsilon^{1-k} H_{+}\right)\right\|_{L^{p}\left(B_{2 R}\right)} .
$$

Therefore,

$$
\int_{B_{2 R}}\left|\eta v^{\prime}\right|^{p} \leqslant C_{4}(F, G, K, p)\left(\int_{B_{2 R}}\left|\left(v-v_{B_{2 R}}\right) \otimes \eta^{\prime}\right|^{p}+\int_{B_{2 R}} \eta\left(\varepsilon+\varepsilon^{1-k} H_{+}\right)^{p}\right),
$$

where $C_{4}(F, G, K, p)=(C(F, G, K, p))^{p}$. Successively using (51) and the Poincaré-Sobolev inequality (for example, see [36, Theorem 4.10.3]), we obtain

$$
\begin{aligned}
& \int_{B_{R}}\left|v^{\prime}\right|^{p} \leqslant C_{5}(F, G, K, p)\left(R^{-p} \int_{B_{2 R}}\left|v-v_{B_{2 R}}\right|^{p}+\int_{B_{2 R}} \eta\left(\varepsilon+\varepsilon^{1-k} H_{+}\right)^{p}\right) \\
& \leqslant C_{6}(F, G, K, p)\left(R^{-p}\left(\int_{B_{2 R}}\left|v^{\prime}\right|^{\frac{n p}{n+p}}\right)^{\frac{n+p}{n}}+\int_{B_{2 R}}\left(\varepsilon+\varepsilon^{1-k} H_{+}\right)^{p}\right)
\end{aligned}
$$

with some consants $C_{5}(F, G, K, p)$ and $C_{6}(F, G, K, p)$. Therefore,

$$
\frac{1}{\left|B_{R}\right|} \int_{B_{R}}\left|v^{\prime}\right|^{p} \leqslant C_{7}(F, G, K, p)\left(\left(\frac{1}{\left|B_{2 R}\right|} \int_{B_{2 R}}\left|v^{\prime}\right|^{\frac{n p}{n+p}}\right)^{\frac{n+p}{n}}+\frac{1}{\left|B_{2 R}\right|} \int_{B_{2 R}}\left(\varepsilon+\varepsilon^{1-k} H_{+}\right)^{p}\right) .
$$

Hence, we have the reverse Hölder inequality

$$
\begin{aligned}
\left(\frac{1}{\left|B_{R}\right|} \int_{B_{R}}\left|v^{\prime}\right|^{p}\right)^{\frac{n}{n+p}} \leqslant \frac{C_{8}(F, G, K, p)}{\left|B_{2 R}\right|} \int_{B_{2 R}}\left|v^{\prime}\right|^{\frac{n p}{n+p}} & \\
& +\left(\frac{1}{\left|B_{2 R}\right|} \int_{B_{2 R}}\left(C_{9}(F, G, K, p)\left(\varepsilon+\varepsilon^{1-k} H_{+}\right)\right)^{p}\right)^{\frac{n}{n+p}} .
\end{aligned}
$$

Put $q=\frac{n+p}{n}>1, f=\left|v^{\prime}\right|^{\frac{n p}{n+p}}$, and $g=\left(C_{9}(F, G, K, p)\left(\varepsilon+\varepsilon^{1-k} H_{+}\right)\right)^{\frac{n p}{n+p}}$. By Lemma 3.4 we conclude that $f$ is integrable with a power slightly larger than $q$. This in turn means that $v^{\prime}$ is integrable with a slightly higher power then $p$ and so $v \in W_{\text {loc }}^{1, p^{\prime}}\left(V ; \mathbb{R}^{m}\right)$ for some $p^{\prime}>p$. $\triangleright$

Acknowlegement. The author is greteful to A. P. Kopylov, Yu. G. Reshetnyak, A. S. Romanov, and S. K. Vodop'yanov for helpful discussions.

\section{References}

1. Astala K. Area distortion of quasiconformal mappings // Acta Math.-1994.-Vol. 173, № 1.-P. 37-60.

2. Astala K., Iwaniec T., Martin G. Elliptic Partial Differential Equations and Quasiconformal Mappings in the Plane.-Princeton: Princeton Univ. Press, 2009. - xvi +677 p.-(Princeton Mathematical Series. Vol. 48.).

3. Ball J. M. Convexity conditions and existence theorems in nonlinear elasticity // Arch. Ration. Mech. Anal.-1977.--Vol. 63.-P. 337-403. 
4. Ball J. M. Constitutive inequalities and existence theorems in nonlinear elastostatics // Nonlinear Analysis and Mechanics: Heriot-Watt Symposium (Edinburgh, 1976).-London: Pitman, 1977.Vol. 1.-P. 187-241.

5. Ball J. M., Currie J. C., Olver P. J. Null Lagrangians, weak continuity, and variational problems of arbitrary order // J. Funct. Anal.-1981.-Vol. 41.-P. 135-174.

6. Belinskī̌ P. P. General Properties of Quasiconformal Mappings.-Novosibirsk: Nauka, 1974.-[Russian].

7. Bezrukova O. L., Dairbekov N. S., Kopylov A. P. On mappings which are close in the $C$-norm to classes of solutions of linear elliptic partial differential equations // Tr. Inst. Math.-1987.-Vol. 7.-P. 1930.-[Russian].

8. Bojarski B. Homeomorphic solutions of Beltrami systems // Dokl. Akad. Nauk SSSR.-1955.Vol. 102.-P. 661-664.-[Russian].

9. Bojarski B. Generalized solutions of a system of first-order differential equations of elliptic type with discontinuous coefficients // Math. Sb.-1957.-Vol. 43 (85), № 4.-P. 451-503.-[Russian].

10. Bojarski B., Gutlyanskii V., Martio O., Ryazanov V. Infinitesimal Geometry of Quasiconformal and bi-Lipschitz Mappings in the Plane.-Zurich: European Math. Soc., 2013.-ix +205 p.

11. Dairbekov N. S. Stability in the $C$-Norm of Classes of Solutions of Linear Elliptic Partial Differential Equations. Candidate's dissertation.-Novosibirsk, 1986.-[Russian].

12. Dairbekov N. S. The concept of a quasiregular mapping of several $n$-dimensional variables // Dokl. Akad. Nauk, Ross. Akad. Nauk.-1992.-Vol. 324, № 3.-P. 511-514. [Russian]; Engl. transl.: Russ. Acad. Sci., Dokl., Math.-1992.-Vol. 45, № 3.-P. 578-582.

13. Dairbekov N. S. Quasiregular mappings of several $n$-dimensional variables // Sib. Mat. Zh. -1993 . Vol. 34, № 4.-P. 87-102.-[Russian]; Engl. transl.: Sib. Math. J.-1993.-Vol. 34, № 4.-P. 669-682.

14. Dairbekov N. S. Stability of Classes of Mappings, Beltrami Equations, and Quasiregular Mappings of Several Variables. Doctoral dissertation.-Novosibirsk, 1995.-[Russian].

15. Edelen D. G. B. Non Local Variations and Local Invariance of Fields.-N. Y.: Elsivier, 1969.-(Modern Anal. and Comp. Methods in Sci. and Engineering. Vol. 19.).

16. Egorov A. A. Stability of classes of solutions to partial differential relations constructed by convex and quasiaffine functions // Proc. on geometry and analysis.-Novosibirsk: Izdatel'stvo Instituta Matematiki SO RAN, 2003.-P. 275-288.-[Russian].

17. Egorov A. A. Stability of classes of solutions to partial differential relations constructed by quasiconvex functions and null Lagrangians // Equadiff 2003.-Hackensack: World Sci. Publ., 2005.-P. 1065-1067.

18. Egorov A. A. Stability of classes of mappings, quasiconvexity, and null Lagrangians // Dokl. Akad. Nauk, Ross. Akad. Nauk.-2007.-Vol. 415, № 6.-P. 730-733.-[Russian]; Engl. transl.: Dokl. Math. 2007.-Vol. 76, № 1.-P. 599-602.

19. Egorov A. A. Quasiconvex functions and null Lagrangians in the stability problems of classes of mappings // Sib. Mat. Zh.-2008.-Vol. 49, № 4.-P. 796-812.-[Russian]; English transl.: Sib. Math. J.-2008.-Vol. 49, № 4.-P. 637-649.

20. Egorov A. A. Solutions of the Differential Inequality with a Null Lagrangian: Regularity and Removability of Singularities.-2010.-URL: http://arxiv.org/abs/1005.3459.

21. Egorov A. A. One integral inequality for solutions of the differential inequality with a null Lagrangian and its application // The collection of the scientific articles of the International school-seminar «Lomonosov's readings in Altai 2012» (Barnaul, 20-23 November, 2012).-Barnaul: ASPA, 2012.Vol. 1.-P. 284-289.

22. Egorov A. A. Solutions of the Differential Inequality with a Null Lagrangian: Higher Integrability and Removability of Singularities. II.-(To appear.).

23. Faraco D., Zhong $X$. A short proof of the self-improving regularity of quasiregular mappings // Proc. Amer. Math. Soc.-2006.-Vol. 134, № 1.-P. 187-192.

24. Gao H. Regularity for weakly $\left(K_{1}, K_{2}\right)$-quasiregular mappings // Science in China. Ser. A. - 2003.Vol. 46, № 4.-P. 499-505.

25. Gao H., Huang Q., Qian F. Regularity for weakly $\left(K_{1}, K_{2}(x)\right)$-quasiregular mappings of several $n$-dimensional variables // Front. Math. China.-2011.-Vol. 6, № 2.-P. 241-251.

26. Gao H., Li T. On degenerate weakly $\left(K_{1}, K_{2}\right)$-quasiregular mappings // Acta Math. Sci. Ser. B.2008.-Vol. 28, № 1.-P. 163-170.

27. Gao H., Zhou S., Meng Y. A new inequality for weakly $\left.\left(K_{1}, K_{2}\right)\right)$-quasiregular mappings // Acta Math. Sin. Engl. Ser.-2007.-Vol. 23, № 12.-P. 2241-2246.

28. Gehring $F$. $W$. The $L^{p}$-integrability of the partial derivatives of a quasiconformal mapping // Bull. Am. Math. Soc.-1973.-Vol. 79.-P. 465-466. 
29. Gol'dshtein V. M. The behavior of mappings with bounded distortion when the coefficient of distortion is close to unity // Sib. Mat. Zh.-1971.-Vol. 17, № 6.-P. 1250-1258.-[Russian]; English transl.: Sib. Math. J.-1971.-Vol. 17, № 6.-P. 900-906.

30. Gol'dshtein V. M., Reshetnyak Yu. G. Introduction to the Theory of Functions with Generalized Derivatives, and Quasiconformal Mappings.-M.: Nauka, 1983.-285 p.-[Russian].

31. Gol'dshtein V. M., Reshetnyak Yu. G. Quasiconformal Mappings and Sobolev Spaces.-Dordrecht etc.: Kluwer Acad. Publ., 1990.-xix+371 p.-(Math. and its Appl. Soviet Ser. Vol. 54.).

32. Gutlyanskii V., Ryazanov V., Srebro U., Yakubov E. The Beltrami Equation. A Geometric Approach.Berlin: Springer, 2012.-xiii +301 p.-(Developments in Math. Vol. 26.).

33. Iwaniec T. On $L^{p}$-integrability in PDE's and quasiregular mappings for large exponents // Ann. Acad. Sci. Fenn. Ser. AI.-1982.-Vol. 7.-P. 301-322.

34. Iwaniec T. p-Harmonic tensors and quasiregular mappings // Ann. Math.-1992.-Vol. 136.-P. 651-685.

35. Iwaniec T., Martin G. Quasiregular mappings in even dimensions // Acta Math.-1993.-Vol. 170.P. 29-81.

36. Iwaniec T., Martin G. Geometric Function Theory and Non-Linear Analysis. Oxford Math. Monogr.Oxford: Oxford Univ. Press, 2001.

37. Iwaniec T., Migliaccio L., Nania L., Sbordone C. Integrability and removability results for quasiregular mappings in high dimensions // Math. Scand.-1994.-Vol. 75, № 2.-P. 263-279.

38. Knops R. J., Stuart C. A. Quasiconvexity and uniqueness of equilibrium solutions in nonlinear elasticity // Arch. Ration. Mech. Anal.-1984.-Vol. 86, № 3.-P. 233-249.

39. Kopylov A. P. Stability of classes of multidimensional holomorphic mappings. III. Properties of mappings that are close to holomorphic mappings // Sib. Mat. Zh.-1983.-Vol. 24, № 3.-P. 7091.-[Russian]; English transl.: Sib. Math. J.-1983.-Vol. 24, № 3.-P. 373-391.

40. Kopylov A. P. Stability of Classes of Multidimensional Holomorphic Mappings. Doctoral dissertation.Novosibirsk, 1984.-[Russian].

41. Kopylov A. P. Stability in the $C$-Norm of Classes of Mappings.-Novosibirsk: Nauka, 1990.-[Russian].

42. Kopylov A. P. On stability of classes of conformal mappings. I // Sib. Mat. Zh. - 1995.-Vol. 36, № 2.-P. 348-369.-[Russian]; English transl.: Sib. Math. J.-1995.-Vol. 36, № 2.-P. 305-323.

43. Kopylov A. P. On stability of classes of conformal mappings. III // Sib. Mat. Zh. - 1997.-Vol. 38, № 4.-P. 825-842.-[Russian]; English transl.: Sib. Math. J.-1997.-Vol. 38, № 4.-P. 715-729.

44. Kristensen J. Finite functionals and Young measures generated by gradients of Sobolev functions. Math. Inst. Technical Univ. of Denmark Mat-Report № 1994-34.-Denmark: Lyngby, 1994.-58 p.

45. Krushkal' S. L. Quasiconformal Mappings and Riemann Surfaces.-Novosibirsk: Nauka, 1975.-195 p.[Russian].

46. Krushkal' S. L. Quasiconformal Mappings and Riemann Surfaces. Scripta Series in Math. A Halsted Press Book.-Washington: V. H. Winston \& Sons, N. Y. etc.: John Wiley \& Sons, 1979.-xii +319 p.

47. Landers $A$. W. Invariant multiple integrals in the calculus of variations // Contributions to the Calculus of Variations, 1938-1941.-Chicago: Univ. of Chicago Press, 1942.-P. 184-189.

48. Lehto $O$. Remarks on the integrability of the derivatives of quasiconformal mappings // Ann. Acad. Sci. Fenn. Ser. AI.-1965.-Vol. 371.-P. 1-8.

49. Lehto O., Virtanen K. I. Quasiconformal Mappings in the Plane. 2nd ed. Grundlehren der Math. Wissenschaften. Vol. 126.-Berlin-Heidelberg-N. Y.: Springer-Verlag, 1973.-viii+258 p.

50. Martio O., Rickman S., Väisälä J. Topological and metric properties of quasiregular mappings // Ann. Acad. Sci. Fenn. Ser. AI.-1971.-Vol. 488.-31 p.

51. Martio $O$. On the integrability of the derivative of a quasiregular mapping // Math. Scand.-1974.Vol. 35.-P. 43-48.

52. Martio O. Modern Tools in the Theory of Quasiconformal Maps. Textos de Matematica. Serie B. Vol. 27.-Coimbra: Universidade de Coimbra, Departamento de Matematica, 2000-43 p.

53. Martio O., Ryazanov V., Srebro U., Yakubov E. Moduli in Modern Mapping Theory.-N. Y.: Springer, 2009.-xii $+367 \mathrm{p}$.

54. Meyers N. G., Elcrat A. Some results on regularity for solutions of non-linear elliptic systems and quasi-regular functions // Duke Math. J.-1975.-Vol. 42.-P. 121-136.

55. Morrey Ch. B. Multiple Integrals in the Calculus of Variations. Grundlehren der Math, Wiss. Vol. 130.Berlin etc.: Springer-Verlag, 1966.

56. Müller S. Variational models for microstructure and phase transitions Calculus of variations and geometric evolution problems // Lectures given at the 2nd session of the Centro Internazionale Matematico Estivo (CIME), Cetraro, Italy, June 15-22, 1996.-Berlin: Springer, 1999.-P. 85-210.(Lect. Notes Math. Vol. 1713). 
57. Reshetnyak Yu. G. Stability estimates in Liouville's theorem and $L_{p}$-integrability of the derivatives of quasiconformal mappings // Sib. Mat. Zh.-1976.-Vol. 17, № 4.-P. 868-896.-[Russian]; English transl.: Sib. Math. J.-1976.-Vol. 17, № 4.-P. 653-674.

58. Reshetnyak Yu. G. Space Mappings with Bounded Distortion.-Novosibirsk: Nauka, 1982.-288 p.[Russian].

59. Reshetnyak Yu. G. Stability Theorems in Geometry and Analysis.-Novosibirsk: Nauka, 1982.-232 p.[Russian].

60. Reshetnyak Yu. G. Space Mappings with Bounded Distortion.-Providence (R. I.): Amer. Math. Soc., 1989.-362 p.-(Transl. of Math. Monogr. Vol. 73.).

61. Reshetnyak Yu. G. Stability Theorems in Geometry and Analysis.-Dordrecht: Kluwer Acad. Publ., 1994.-xi+394 p.-(Math. and its Appl. Vol. 304.).

62. Reshetnyak Yu. G. Stability Theorems in Geometry and Analysis. 2nd rev. ed.-Novosibirsk: Izdatel'stvo Instituta Matematiki SO RAN, 1996.-424 p.-[Russian].

63. Rickman S. Quasiregular Mappings.-Berlin: Springer-Verlag, 1993.-x+213 p.-(Results in Math. and Related Areas (3). Vol. 26.).

64. Sokolova T. V. Behavior of nearly homothetic mappings // Mat. Zametki.-1991.-Vol. 50, № 4.P. 154-156.-[Russian]; English transl.: Math. Notes.-1991.-Vol. 50, № 4.-P. 1089-1090.

65. Sokolova T. V. Stability in the Space $W_{p}^{1}$ of Homothety Transformations. Candidate's dissertation.Novosibirsk, 1991.-[Russian].

66. Sychev M. Young measure approach to characterization of behaviour of integral functionals on weakly convergent sequences by means of their integrands // Ann. Inst. H. Poincaré Anal. Non Linéaire.1998.-Vol. 15.-P. 755-782.

67. Tong Y., Gu J. Li Y. A new inequality for weakly $\left(K_{1}, K_{2}\right)$-quasiregular mappings // J. Inequal. Pure Appl. Math.-2007.-Vol. 8, № 3, Paper № 91.-4 p.

68. Väisälä J. Lectures on $n$-Dimensional Quasiconformal Mappings.-Berlin-Heidelberg-N. Y.: SpringerVerlag, 1971.-xiv +144 p.

69. Vuorinen M. Conformal Geometry and Quasiregular Mappings.-Berlin etc.: Springer-Verlag, 1988.$x i x+209$ p.-(Lect. Notes Math. Vol. 1319.).

Received April 3, 2013.

Egorov Alexander Anatol'evich

Sobolev Institute of Mathematics,

senior researcher

RUSSIA, 630090, Novosibirsk, Koptyug Avenue, 4;

Novosibirsk State University,

associate professor

RUSSIA, 630090, Novosibirsk, Pirogova Str., 2

E-mail: yegorov@math.nsc.ru

\title{
РЕШЕНИЯ ДИФФЕРЕНЦИАЛЬНОГО НЕРАВЕНСТВА С НУЛЬ-ЛАГРАНЖИАНОМ: ПОВЫШАЮШАЯСЯ ИНТЕГРИРУЕМОСТЬ И УСТРАНИМОСТЬ ОСОБЕННОСТЕЙ. І
}

\author{
Егоров А. А.
}

\begin{abstract}
Целью настоящей статьи является установление свойства самоулучшающейся интегрируемости производных решений дифференциального неравенства с нуль-лагранжианом. Более точно, мы доказываем, что решение класса Соболева с показателем суммирумости, немного меньшим естественно определенного структурными предположениями на нуль-лагранжиан показателя, фактически принадлежит пространству Соболева с показателем суммируемости, немного большим естественного показателя. Мы также применяем это свойство, чтобы улучшить теоремы о гёльдеровой регулярности и об устойчивости из статьи [19].
\end{abstract}

Ключевые слова: нуль-лагранжиан, повышающаяся интегрирумость, самоулучшающаяся регулярность, гёльдерова регулярность, устойчивость классов отображений. 